San Jose State University

SJSU ScholarWorks

Faculty Research, Scholarly, and Creative Activity

$12-13-2018$

\title{
The relationship between polychronicity and social networks: A mixed-methods study of research and development professionals
}

\author{
Fabiola Bertolotti \\ University of Modena and Reggio Emilia \\ Elisa Mattarelli \\ San Jose State University, elisa.mattarelli@sjsu.edu \\ Janet Dukerich \\ University of Texas at Austin
}

Follow this and additional works at: https://scholarworks.sjsu.edu/faculty_rsca

Part of the Business Administration, Management, and Operations Commons

\section{Recommended Citation}

Fabiola Bertolotti, Elisa Mattarelli, and Janet Dukerich. "The relationship between polychronicity and social networks: A mixed-methods study of research and development professionals" Human Relations (2018): 1595-1622. https://doi.org/10.1177/0018726718810097

This Article is brought to you for free and open access by SJSU ScholarWorks. It has been accepted for inclusion in Faculty Research, Scholarly, and Creative Activity by an authorized administrator of SJSU ScholarWorks. For more information, please contact scholarworks@sjsu.edu. 


\title{
The Relationship between polychronicity and social networks:
}

\section{A mixed-method study of research and development professionals}

\author{
Fabiola Bertolotti, Elisa Mattarelli and Janet Dukerich
}

\begin{abstract}
How do knowledge workers interact with their colleagues when organizations increasingly ask them to work on multiple activities, projects, and working spheres simultaneously? Given the importance of social networks for individual and organizational success, in this study we explore the relationship between individual preferences for engaging in multiple tasks simultaneously (individual polychronicity), the perception of the organization's demands in terms of engaging in multiple tasks simultaneously (organizational polychronicity), and centrality in instrumental networks. Adopting a mixed methods approach, we collected data from knowledge professionals in a research and development (R\&D) unit. Our results show that both individual and organizational polychronicity were related to network centrality. However, the effect of individual polychronicity on instrumental network centrality was stronger, especially for advice-related interactions, suggesting that individual preferences matter more when it comes to knowledge related interactions. Not only we link polychronicity to a previously unexplored context, i.e. social networks, but we also propose the use of a cultural tool kit perspective to explain how individuals differentially make sense of organizational temporal demands. Finally, we advance research on the antecedents of network centrality and contribute to the ongoing debate on the delicate balance between structure and individual characteristics.
\end{abstract}

\section{Keywords}

agency, centrality, instrumental social networks, individual polychronicity, organizational polychronicity, structure, cultural tool kit, time 


\section{Introduction}

Researchers have long been interested in understanding the processes by which individuals' relationships develop in organizations (e.g. Brass et al. 2004; Granovetter, 1985; Kilduff and Tsai, 2003). The networks that individuals build, above and beyond what is formally asked to them (e.g. when employees are required to work with colleagues in project teams), are relevant not only for their immediate outcomes but also for organizational success (Kilduff and Brass, 2010). For instance, we know that knowledge and other valuable workrelated resources such as task-advice, strategic information, and social support (e.g. Brass, 1984; Ibarra, 1993; Sparrowe, et al., 2001) flow mainly through workplace social networks. Because networks are interpersonal phenomena (Landis, 2016) their development has been associated - in different research streams - to organizational context variables. In particular, structure and formal division of labor are supposed to provide both opportunities and constraints in interacting with others (e.g. Shrader et al. 1989). In addition, actors' characteristics such as self-monitoring or extraversion (personality traits which are thought to affect sociality) influence how many relationships an individual develops in the workplace (e.g. Asendorpf and Wilpers, 1998; Oh and Kilduff, 2008).

In the interest to advance our understanding of the person vs situation debate in network studies, we are now witnessing a renewed attention to the integration of psychological and network perspectives in organizational research to appraise how workers' individual differences may influence their tendency to interact with others in the workplace (e.g. Bensaou et al. 2014; Casciaro, et al., 2015; Fang et al., 2015; Kleinbaum et al., 2015; Landis, 2016). Given the widespread conviction that social networks offer great opportunities to people but require significant investments of energy and time to be maintained (Day and Kilduff, 2003; Landis, 2016), time-based individual differences seem likely to play a relevant role in shaping the nature of relationships or one's position in workplace social networks. 
However, the question of whether time-related individual differences offer a basis for understanding why some individuals may occupy central positions has not been explored, limiting the development of fuller accounts of the actors' agency in crafting their social networks, within organizational constraints.

Organizations increasingly ask their employees to work on multiple tasks simultaneously and to juggle between different team projects (O'Leary et al., 2011), activities (Cotte and Ratneshwar, 1999), roles (Carton and Ungureanu, 2018) and working spheres (Gonzales and Mark, 2004) exposing individuals to potential interactions with a larger set of colleagues than ever before. In this paper we concentrate on one specific time-based individual characteristic that reflects the preference or desire to work on different tasks in the same block of time, or polychronicity (Bluedorn et al., 1999). Individuals may vary greatly in terms of their preferences for focusing on one task at a time (highly monochronic) as opposed to working on many things at once (highly polychronic) and their beliefs about what is the best way to work. Polychronicity thus refers to preferences about sequencing activities and reflects how workers prefer to allocate a precious resource like their work time (Soutaris and Maestro, 2010). Specifically, we argue that these preferences might influence not only what and how many tasks they focus on, but who and how many different individuals they may interact with in getting the work done.

Previous research on polychronicity suggests that time-use orientations influence interpersonal behaviors and that polychronic individuals are more likely to prefer interpersonal relationships over task demands than monochronic individuals (e.g. Bluedorn, 2002; Hall, 1959, 1983; Mohammed and Harrison, 2013). Put differently, monochronic individuals interpret activities and events falling outside of the focal task (e.g. an unexpected request from a coworker) as interferences to be avoided while for polychronic individuals these activities are part of the normal way of working. Consequently, it is arguable that 
monochronic and polychronic individuals will hold different awareness and interpretations of the investments in energy and time entailed in building and maintaining large networks. We thus propose that individual polychronicity will be related to the ongoing social relationships in which organizational actors are embedded in the workplace. Specifically, we theorize that polychronic individuals will occupy a more central position in the instrumental workplace social networks than their monochronic counterparts.

Monochronic and polychronic differences may also refer to individuals' perceptions about their organization's preferences in terms of sequencing and organizing work activities. Some organizations may convey norms and values that accentuate polychronic preferences while others could suggest practices or even create rituals consistent with monochronic preferences (e.g. Stephens et al., 2012). In addition, organizations place different demands on workers through the assignment on projects and work activities. In this paper we build on Swidler's (1986) perspective of culture as a 'tool kit' to explain how workers, drawing differently on the time-based resources provided by the organization (stories, rituals, and practices) may develop their own perception of the organizational polychronicity and construct accordingly their actions in particular situations, especially in relation to centrality in workplace social networks. Thus, we argue, individuals' perceptions about their organization's preferences for engaging in multiple tasks simultaneously may relate to their willingness to nurture work related relationships, influencing their central positions in the instrumental networks.

We also argue that the beliefs about the organization's preferences will be more strongly related to instrumental network centrality than individual preferences.

We explore the relationship between individual and perceived organizational polychronicity and centrality in instrumental social networks in the context of knowledge professionals engaged in $R \& D$ activities and operating in a multi-project context. We adopted 
a mixed methods research design (Creswell, 2003) and collected both quantitative data to test the relationship between individual and perceived organizational polychronicity and social network centrality, and qualitative data to explore more deeply how professionals made sense of their individual preferences and what they believed the organization expected from them.

The present research intends to make several contributions. By investigating the relationship between individual and perceived organizational polychronicity and the enactment of instrumental social networks we extend research on polychronicity linking it to unexplored variables such as instrumental networks size. This is an important link to address as time is not only a matter of how tasks are perceived but also of how people in organizations coordinate their relationships to deal with the tasks (e.g. Bluedorn et al. 1992). In addition, drawing on Swidler's theory of cultural tool kits we contribute to a more nuanced understanding of the construct of organizational polychronicity. In contrast to previous studies (e.g. Slocombe and Bluedorn, 1999), we suggest that even within the same work unit, individuals may experience some variability in terms of their perception of organizational polychronicity as a consequence of drawing differently from the various cultural tool kits proposed to them. Finally, by showing how perceptions of situational factors (organizational polychronicity) and time-related individual traits (individual polychronicity) shape instrumental network structures we discuss the relative importance of personality variables, organizational variables, and structural variables. Thus, we contribute to the ongoing debate in network research on the weight of structure versus individual characteristics and cognitions in shaping social networks (see Bensaou et al. 2014; Emirbayer and Goodwin, 1994; Emirbayer and Mische, 1998; Tasselli et al., 2015). 


\section{Theory and hypothesis development}

\section{Individual polychronicity and interpersonal relationships}

Polychronicity is an individual difference that reflects the extent to which individuals prefer to be involved concurrently in more than one task and believe their preference is the best way of doing things (Bluedorn et al., 1999; Bluedorn, 2002). Polychronicity is conceptualized on a continuum, so that the more individuals prefer being engaged in, and alternating among, different tasks, the more they are polychronic.

Two elements of the definition of polychronicity deserve attention. First, the term 'concurrently' refers to two possible situations, i.e. sequentially accomplishing tasks and simultaneously accomplishing tasks (Stephens et al., 2012). In the former case, a person, engaged in multiple tasks (say A, B, and C), enacts an 'intermittent pattern - resume A from a previous time, stop A and begin B, stop B and begin A, stop A and begin C, stop C and return to A' (Bluedorn, et al., 1992, p. 17). The latter case is exemplified by a person who 'is writing a letter, talking on the phone, eating an apple, and listening to the War of 1812 Overture' all at the same time (Bluedorn, et al., 1992, p. 18).

Second, the degree of polychronicity is a habitual preference or belief factor in multiple task environments and it is thought to be a relatively stable trait of an individual. In other words, the concept relates to people's beliefs and preferences that are assumed to manifest in polychronic behaviors (e.g. frequent switching back and forth between activities and scheduling of two or more activities at a time, Slocombe and Bluedorn, 1999).

Several empirical studies have examined the relationship between polychronicity and individual characteristics and outcomes. For instance, scholars investigating the Big Five personality dimensions found that agreeableness, neuroticism and openness seem not related to polychronicity. However, polychronic people tend to be less conscientious and more extroverted (Conte and Jacobs, 2003; Conte and Gintoft, 2005; König et al., 2005; Payne and 
Philo 2002). In addition, Kaufman, Lane and Lindquist (1991) showed that polychronicity was positively related to educational level and average number of working hours per week and negatively related to the perception of role overload. Hall and Hall (1990) observed that polychronic people were more flexible with changes in plans and had a higher information retention capacity. Other researchers found a relationship between polychronicity and increased job satisfaction (Arndt et al., 2006; Jang and George, 2012) as well as increased perceptions of frustration and confusion in the workplace (Cotte and Ratneshwar, 1999). Results regarding the relationship between polychronicity and individual job performance are mixed (e.g. Conte and Gintoft, 2005; Conte and Jacobs, 2003; Payne and Philo, 2002). The influence of polychronicity on job performance may depend on the nature of work such that the relationship will be positive when the work environment requires multitasking (König and Waller, 2010).

Particularly relevant for our study is the link between polychronicity and interpersonal relationships in the workplace. On the one hand, monochronic people 'look at time as linear and separable, capable of being divided into units' (Cotte and Ratneshwar, 1999: 186) and prefer scheduling everything allowing only few unexpected events within a given period (Hall, 1983). Thus, for monochronic individuals, adherence to schedule drives the setting of priorities for both activities and people. When working on a specific task or project, events that drive attention away from the same project such as, for instance, a colleague stopping by the office without appointment with questions related to different projects, are perceived by monochrons as disturbances to be avoided (Bluedorn, 2002; Mohammed and Harrison, 2013).

On the other hand, polychronic individuals 'look at time as naturally re-occurring and consequently behave by using time for many purposes at once' (Cotte and Ratneshwar, 1999: 189). Their preference for working on several tasks simultaneously makes them feel comfortable with unscheduled events that are perceived as normal and squeezed into their 
existing work patterns (Bluedorn et al., 1999) and leads them to be continually involved with others and interact with several people at once (Hall, 1983). Because a polychronic time preference emphasizes being available for emergent requests from the task or other people rather than adherence to schedules, polychrons may accept interruptions by others more than monochrons.

\section{Individual polychronicity and centrality in the instrumental network}

The interactive activities discussed above can be understood and represented as social networks (Wasserman and Faust, 1994). Research on social networks in the workplace has investigated different types of instrumental interactions (e.g. communication, advice, knowledge transfer, Bertolotti et al., 2005; Cross and Cummings, 2004; Sykes et al., 2014; Tagliaventi and Mattarelli, 2006) and the importance for individuals to occupy central network positions (e.g. Ahuja et al., 2003; Ibarra, 1993; Sparrowe et al., 2001). Network centrality (i.e. the number of people in the network someone is directly tied to) has been associated to positive outcomes for individuals like enhanced work-related resources, including task advice and quality and quantity of strategic information, that often result in improved job performance (Baldwin et al., 1997; Cross and Cummings, 2004; Fang et al., 2015; Mehra et al., 2001; Sparrowe et al., 2001; Sykes et al. 2014).

Given the importance of individual centrality in social networks, scholars have paid considerable attention to its antecedents, both in terms of individual and organizational characteristics. In particular, research demonstrates how the tendency to occupy central positions in social networks is positively related to individual personality differences such as self-monitoring orientation (Mehra et al., 2001; Oh and Kilduff, 2008), conscientiousness (e.g. Lee et al., 2010; Liu and Ipe, 2010), and extraversion (e.g. Asendorpf and Wilpers, 1998; Pollet et al., 2011). Conversely, individuals high in neuroticism tend to occupy more 
peripheral positions (Kalish and Robins, 2006; Klein et al., 2004). Despite these attempts to investigate how individual differences are relevant to social networks, the question of whether time-related individual differences offer a basis for understanding why some individuals may occupy central positions has not been explored. We believe focusing on how this particular individual difference variable affects social networks is important and timely because of the increasing demands placed on employees to work on a multitude of tasks, projects, and teams simultaneously. In order to add to this literature, we propose that individual polychronicity will positively relate to individual centrality in instrumental social networks, i.e. those interactions that are associated to get the work done, such as communicating with colleagues and exchanging advice (Ibarra, 1992). We base our reasoning on the following arguments.

In the complex and often unpredictable context of professionals operating in knowledge intensive environments, it is likely that a large percentage of interactive activities would occur spontaneously and a large proportion of unexpected events falling outside a focal task would be caused by colleagues asking questions or communicating information or necessitating pieces of more or less specific advice (e.g. Perlow, 1999). The monochrons' preferences to shield themselves from those types of interferences in order to keep concentrating on the current task completion could lead them to be unwilling to address many requests from colleagues thus affecting the networks connecting them to others. Conversely, since polychronic individuals prefer being available for emergent requests from the task or other people, and feel comfortable with changes in their activities that they perceive as normal in the workplace, we expect that they will be more willing to address teammates' requests, providing them with the opportunity to be seen as a stable point of reference in the networks by colleagues. 
In addition, given that polychronic people do not perceive time as a quantifiable resource (Ballard and Seibold, 2000), they may have a different interpretation and awareness of the investment in terms of time entailed in sustaining large networks. More specifically, compared to monochrons, polychrons may consider the development of high centrality in network as less daunting.

All the above may lead polychrons to occupy a more central position in the instrumental network than their monochronic counterparts. In other words, they will engage in more work-related communication in the workplace and they will be open to provide advice, suggestions, and knowledge with a higher number of co-workers.

Hypothesis 1: Individual polychronicity is positively related to centrality in the instrumental network.

\section{Perceptions of organizational polychronicity and centrality in the instrumental network}

Organizations, of course, impose 'constraints' on what to work on and how to organize work patterns, and they hold norms for the completion of tasks and how to engage in multiple tasks (Stephens et al., 2012; Mattarelli et al., 2015). Research has shown that polychronicity is also a component of organizational culture. However, individuals may hold different perceptions of the level of organizational polychronicity (Bluedorn et al., 1999). The organizational culture includes a set of frames, rituals, and practices that individuals use to define what are considered acceptable ways to schedule activities in time, influencing organizational behaviors and actions (Blount and Janicik, 2001; Blount and Leroy, 2007). We hypothesize that what individuals perceive about the organization's demands to engage in multiple tasks simultaneously, i.e. the perceived organization's polychronicity, is related to their behaviors and relationships in the organization, i.e. their social networks. 
Consistent with a 'tool kit' perspective (Swidler, 1986, 2000; Kellogg, 2011; Giorgi et al., 2015), we do not consider culture as a unified system of values that unidirectionally causes uniform employees' perceptions and behaviors, but a tool kit of resources that individuals draw from in order to shape their interpretations and actions. According to this perspective, an organizational culture provides 'resources from which people can construct diverse strategies of actions. [...] To construct such a strategy means selecting certain cultural elements $[\ldots]$ and investing them with particular meanings in concrete life circumstances' (Swidler, 1986: 281). Thus, the practices and rituals that organizations put in place in relation to scheduling activities and moving between projects and tasks represent a repertoire that individuals can draw from to interpret organizational preferences and, consequently, inform their behaviors (e.g., Ballard and Seibold, 2003; Ballard and Seibold, 2004).

For instance, some organizations encourage their members to engage in multiple tasks and play multiple roles during a same week or a same day (Ashforth et al., 2000; Wasson, 2004). In other organizations, visible artifacts and methods, such as the Pomodoro technique, are used to communicate that a monochronic use of time is preferable (Gobbo and Vaccari, 2008). Blount and Janicik argued that the interpretation of prevailing temporal agenda, that is, 'the individual actors' perception and construal of the organization's temporal structure'(2001: 570), deduced and chosen from the temporal information generated by the work environment, influences the way individuals organize their routines and work schedules.

It is important to further underscore that when individuals mix and match the cultural tool kits, they may come up with different individual repertoires, i.e., interpretations and combinations of cultural features, even if they belong to the same organizational environment (Weber, 2005). When we apply this idea to the case of temporal norms, this means that organizational members, by making different uses of the cultural tool kits at their disposal, 
may vary in how they see their organization more or less polychronic, with implications for individual outcomes.

Thus, even if organizational cultural tool kits are available to all organizational members, different individual interpretations of culturally based time-related practices may impact on how professionals respond to time demands made by co-workers and interact with them to solve those demands (Blount and Leroy, 2007), influencing, we argue, their position in the instrumental social networks. Those professionals who perceive that their organizations demand them to work in a highly polychronic environment will structure their social networks accordingly. That is, the more individuals believe their organizations demand to engage in multiple tasks simultaneously, the more likely they will be to engage in interactions in the workplace as well as share advice with a higher number of co-workers. Thus, we hypothesize:

Hypothesis 2: The perception of the organization's polychronicity is positively related to centrality in the instrumental network.

Since individuals strive for congruence between their preferences and the behaviors they are required to exhibit in organizations (König and Waller, 2010), there should be a positive correlation between individual polychronicity and the perception of organizational polychronicity. In their study of employed business school graduates, Slocombe and Bluedorn (1999) found a moderate correlation ( $\mathrm{r}=.45)$ between preferred polychronicity and experienced work-unit polychronicity. The authors showed that the presence of congruence was associated with higher levels of organizational commitment, individuals' perceived performance evaluation by their supervisors and co-workers, and individuals' perceived fairness of the performance evaluation. 
However, individuals may find themselves working in organizations that do not fit with their preferences (Hecht and Allen, 2005). That is, highly monochronic individuals may find themselves facing organization's demands for engaging in multiple tasks simultaneously while highly polychronic individuals may be members of several different project teams but may be asked to work more sequentially. In both situations, their personal preferences for engaging in multiple tasks simultaneously may not match what they believe the organization prefers and values. Interesting questions therefore arise as to which effect (individual preferences or beliefs about the organization's preferences) is stronger on the pattern of social interactions. Such questions have been of interest for decades to organizational behavioral scholars trying to bring insights into the person vs situation debate (e.g. Lucas and Donnellan 2009; Judge and Zapata, 2015). While we expect both personal preferences and perceptions of organizational preferences to relate to behaviors that lead to centrality in individuals' social networks, we take a situational perspective and we argue that individuals will be more responsive to the demands they interpret from the organization. On one hand, we recognize that knowledge workers, endowed with a high level of control and autonomy over their work, may exert agency in trying to align the way work is carried out with their personal preferences influencing not only facets of job tasks but also relational choices (e.g. Wrzesniesky and Dutton 2001; Pratt et al. 2006). On the other hand, extant theory suggests that the perceived situational constraints are higher when employees' actions are relevant for a number of stakeholders (Meyer et al., 2010; Judge and Zapata, 2015) which is likely the case for complex knowledge-intensive collaborative environments. In addition to that, organizational members tend to enact behaviors to obtain positive outcomes from their organization. Performing behaviors that are congruent with the organizational orientations or perceived requests can lead to several positive consequences such as appreciation by peers and supervisors, rewards and career advancements as well as higher performance evaluation 
(Hui et al., 2000; Van Scotter et al., 2000). Additionally, while the organizational culture provides a set of tool kits that individuals can mix and match according to their preferences, deviating from the prevailing tool kits is costly for individuals. According to Swidler (1986), the organizational cultural repertoire limits the available strategies of actions, because of 'the high costs of cultural retooling to adopt to new patterns of actions' (284) that are not consistent with the organizational culture. Thus, if the behaviors of individuals are not determined by the temporal organizational norms, they are strongly influenced by how individuals make sense of it, because of the constraints the perceived organizational polychronicity imposes on individuals in terms of available strategies of action.

Thus, we argue that perceived organizational polychronicity will be more strongly related to the patterns of relationships in the organization as compared to individual polychronicity.

Hypothesis 3: The perception of the organization's polychronicity is more strongly related to centrality in the instrumental network than individual polychronicity.

\section{Methods}

\section{Data collection}

We conducted a mixed methods study in a division of a world-leading business operating in the eco-sustainable mobility industry, for which we gave the fictitious name Re.Search. Re.Search is a medium size company where engineers and technicians carry out research and development activities of advanced systems using alternative fuels for motor propulsion. When we started our project, the company was considered one of the most dynamic medium-sized companies in Italy and experienced the best performance among all the companies listed in the same segment of the Italian stock exchange market. Given the 
attention paid by companies, media and society at large to green energies, at the time of the study Re.Search was facing a high demand for products and services in short timeframes. Professionals were formally assigned to multiple simultaneous projects, making the issue of polychronicity particularly salient.

Engineers and technicians worked on two types of different projects: (1) After Market projects, devoted to develop solutions for final customers and (2) Original Equipment Manufacturer projects, dedicated to create subsystems for specific clients. When we gathered our data, 40 projects were active and they lasted on average 13 months $(S D=7.3)$.

To investigate the relationship between individual and perceived organizational polychronicity and the centrality of R\&D professionals in instrumental social networks we adopted a mixed methods approach (Creswell, 2003), that is to say we triangulated the findings of the first method with evidence collected with a second method. Specifically, our study began with a quantitative method to test our hypotheses and was followed by a qualitative method involving semi-structured interviews.

After preliminary interviews with the VP for Human Resources, the VP for Research and Development and four senior managers, we developed a multi-section questionnaire, consisting of established scales. Respondents indicated their preference to use their time polychronically and their perception of the organization's preference. Another section elicited each respondent's instrumental networks. In addition, management gave us a list of open projects at the time of the study and respondents indicated all that involved them. In the last section of the questionnaire, we asked respondents to provide demographic information (gender, age, educational background, organizational position, organizational and professional tenure). In the next paragraphs, we describe the specific measures we used in the study. 
Because top management approved and supported the research, all 83 members of the R\&D unit turned in the questionnaire, but, due to missing data, we included 71 questionnaires in the final analysis. We guaranteed anonymity to our respondents and asked them to send their completed questionnaires directly to us. Seventy of the 71 respondents were male. Their average age was 34 years $(S D=7.3)$ and they had worked with the organization for an average of five years $(S D=5.5)$. Their professional tenure was 11 years $(S D=9.34)$. Our professionals worked in a multiple project team context and, on average, each of them was a member of 5.2 teams simultaneously $(S D=4)$. Fifty-eight of our respondents $(82 \%)$ were based in the headquarters of the organizations, while the remaining were scattered in the offshore R\&D locations based in China, Brazil, and Iran. Offshore professionals were given the questionnaire in English. The headquarters professionals responded to the questionnaire in Italian. To prepare the Italian version of all the scales we adopted a translation backtranslation method and compared the back-translated items with the original items. In case of variations, we discussed and adjusted the Italian items.

It is interesting to notice that Re.Search shows many typical features of medium-sized Italian firms. For instance, people in technical roles were men. Also, even if supervisory responsibilities were formally assigned to professionals with an engineering degree, hightenured technicians (without a degree) did not experience a low status within the organization. On the contrary, they were considered a point of reference because of their precious work experience gained 'in the field' over the years.

While we relied on survey data to test the hypotheses, we also interviewed some R\&D professionals to develop a deeper understanding of the context where they worked and to further interpret our results. More specifically, we used qualitative data to shed light on the quantitative results and provide a more grounded explanation of the social processes happening in the organization under study. We conducted 10 additional open-ended face-to- 
face interviews (Gubrium and Holstein, 2003) with four project managers and six engineers (nine of them were working at the headquarters and one of them was based at the China offshore site). Interviews lasted approximately 1.5 hours and were fully recorded and transcribed. We asked our informants about the nature of their work in the organization, how they sequenced their work tasks and their preferences about engaging in multiple tasks simultaneously, how they defined themselves as professionals, how they described their organization and the demands they perceived it put on them. We asked our informants to provide us with examples from their experience, instead of giving us general descriptions of their work and their organization. Our respondents were also free to pursue, in more detail, interesting themes and comments arising from each interview.

\section{Measures and reliability}

Individual centrality in the instrumental network. To measure the centrality in the instrumental network we collected full network data (Hanneman and Riddle, 2005) on two types of work-related networks frequently investigated in the social network literature: the communication network and the advice network (e.g. Fang et al., 2015). The communication network reveals all the relationships among employees who talk about any work-related matters (Krackhardt and Hanson, 1993), whereas the advice network includes relations through which individuals share more specific assistance and guidance related to problem solving and the completion of work (Sparrowe, et al., 2001; Lomi et al. 2014).

In order to capture the full communication and advice networks, the questionnaire listed in alphabetical order all the respondents. We assessed the communication network by asking our respondents: "Could you please identify those individuals with whom you have had some work-related communication during the past six months and, in each case, estimate 
the intensity of the relations? By communication we mean conversations, meetings, phone calls or any other interactions related to the completion of your work." We used a five-point scale ranging from (1) "not at all”, (3) “weekly", and (5) “many times a day." We dichotomized the communication network at equal/greater than 2 (i.e., monthly communication), because our interest was in knowing how many people a person had interactions with. To assess the individual centrality in the dichotomized communication network, we further symmetrized the communication matrix, using the higher value. This means that we considered a communication interaction existing between actors $i$ and $j$ if $i$ declared to communicate with $\mathrm{j}$ or vice versa. Respondents' centrality was computed as their degree score (Wasserman and Faust, 1994).

Following the work of Ibarra (1993), Sparrowe et al. (2001) and Lomi et al. (2014), we assessed advice relations by asking our respondents: "In the past six months, to whom have you gone for help or advice on work-related matters at least twice?" We labeled the network as advice giving. On the basis of our theorizing about the relationship between individual and perceived organizational polychronicity and their influence on people's willingness to attend to co-workers' requests for advice and assistance, in the advice network we computed the respondents' centrality in terms of their indegree score (Freeman, 1979; Wasserman and Faust, 1994; Borgatti et al., 2002). The indegree in the advice giving network represents the number of colleagues who declared they could count on a focal actor for workrelated advice (that is an objective measure of the amount of advice offered by a focal actor). Nevertheless, to provide a fuller account of the influence of polychronicity on network patterns, we also computed the outdegree score in the advice giving network that measures how many colleagues a focal individual declares to go for assistance, help or advice. As a post-hoc analysis we present and comment also on the results using the advice outdegree score as our independent variable. 
Individual polychronicity. We assessed our respondents' preference for engaging in multiple tasks at a time using the Polychronic Attitude index (Kaufman et al., 1991; Bluedorn et al., 1992) which consists of four items (see Appendix A). A high score indicates that an individual is more polychronic, while a low score indicates that an individual is more monochronic. We measured the items along a 7-point Likert scale (Cronbach's alpha $=$ $0.73)$

The perception of the organization's polychronicity. We asked each respondent to think about how the work was done in the organization and we used the scale developed and validated by Bluedorn et al. $(1992,1999)$ to assess the organization's perceived polychronicity. Consistent with other studies (e.g. Souitaris and Maestro, 2010) we measured the extent to which the organization was perceived to be polychronic using a concise five-item version of the scale (Bluedorn et al., 1992). Each item (see Appendix A) was scored on a 7-point Likert scale with $7=$ strongly agree and $1=$ strongly disagree $($ Cronbach's alpha $=0.75)$.

Control variables. Because past research has explored other antecedents of network centrality (e.g. Ibarra, 1993), we included several control variables in our model. Past research suggests that organizational tenure can affect network centrality (Mehra et al., 2001; Pappas and Wooldridge, 2007). Thus, we measured organizational tenure in years.

Our professionals varied in the extent to which they had supervisory responsibilities over coworkers and their activities. It was therefore important to control for their formal organizational position because supervisors often assign workload, clarify task assignments, and need to coordinate and support coworkers. Therefore, they can be embedded in more 
dense communication and advice networks. Position was coded as a two-level variable $(0=$ respondent does not supervise anyone; $1=$ respondent supervises some coworkers).

Some of our informants were based at the headquarters of the organization while some were based at offshore locations. Given that being at the headquarters, as opposed to offshore locations, can give access to more contacts for gaining information and knowledge (e.g. Mattarelli and Tagliaventi, 2010), we controlled also for the location of the respondent. Location was coded as a two-level variable $(1=$ respondent is at the headquarter; $0=$ respondent is at an offshore location).

We also added two control variables related to the work individuals performed within their specific project teams. Individuals were assigned to multiple teams simultaneously and, thus, were formally asked to work and collaborate with multiple colleagues. Professionals pulled into projects with a high number of co-workers could need to enact more interactions, regardless of their preferences. To control for the number of formal interactions (the 'required' network), we computed the number of different colleagues a person was formally assigned to work with on all his projects at the time of the study.

Finally, the level of complexity of the work conducted by individuals could also impact on how many interactions they enact. People working on more complex projects may need to get involved in information and knowledge exchanges from multiple sources. In our context, projects differed in terms of complexity. Original Equipment Manufacturing projects (OEM) entailed the development of new and innovative products. Conversely, After Market projects (AM) were mainly devoted to customize already existing products, and were considered of lower complexity. Projects entailing both OEM and AM features were deemed as the most complex. Thus, project complexity was coded as a three-level variable (1=AM; $2=\mathrm{OEM} ; 3=\mathrm{AM} / \mathrm{OEM})$. For each individual we computed the average level of complexity of the projects the person was involved with. 


\section{Data analysis}

Quantitative data analysis. To test our hypotheses, we used UCINET (Borgatti et al., 2002) for nodal regression analysis. Traditional OLS regression analysis could not be performed on our data and on social network data more generally, because of network autocorrelation, i.e. individuals' network data were not independent. The slope coefficients are determined in the same way as for a regression but the testing for significance is done using a permutation test. The algorithm first determines the slope coefficients for a regression and next statistics are recomputed over a large number (1000) of repetitions 'in which covariates are randomly redistributed among respondents, while keeping the topology of the network - and any interdependencies therein - fully intact. The p-value for each statistic is the proportion of permutations that yielded a statistic as extreme as the one initially produced' (Lewis et al., 2008: 335).

Qualitative data analysis. In analyzing the interviews, we wanted to find support and further explanations for the quantitative results, both expected and unexpected. To code the interviews transcripts, we availed ourselves of the coding techniques described by Miles and Huberman (1994) and we followed a two-stage process. We first created a basic 'coding scheme' with the categories included in our hypotheses (individual polychronicity, perceived organizational polychronicity, and networks). To increase the reliability of our analysis, two of us read all the interviews several times to gain a comprehensive overview of the data. Drawing on similar statements, we traced back different phenomena contained in the transcripts to the categories in our coding scheme (e.g., Bertolotti and Tagliaventi, 2007). In addition to using the coding scheme, we also performed an open coding and added themes emerging as the analysis proceeded. Furthermore, we looked for evidence to support the links between the categories (e.g. evidence supporting that individual polychronicity was related to 
centrality in instrumental networks). The first two authors met few times to discuss their individual analysis and reconciled disagreements and thus finalized the coding scheme. In a second stage, we tested the construct validity of the coding scheme. Two of the authors recoded all the evidence using a software to compute inter-rater reliability. Cohen's Kappa, a statistic that adjusts rater agreement to control for chance (Cohen, 1960), was 0.92, indicating high levels of reliability. Again, all disagreements were reconciled through discussion.

To better highlight how the qualitative results illuminate the quantitative relationships, we organized the results section as follows: we present the quantitative analysis for each hypothesis first and then we provide the related qualitative evidence from the interviews.

\section{Results}

Table 1 presents a correlation matrix and descriptive statistics for all the variables used in the models reported next. The table, in line with the literature on polychronicity, shows a significant correlation between individual and perceived organizational polychronicity $(\mathrm{r}=0.45 ; \mathrm{p}<.01)$. It is interesting to underscore that the number of colleagues a person is formally required to work with is positively related to the perception of organizational polychronicity and to network centrality (measured as degree and indegree in the communication and advice networks respectively). In other words, the formal organizational structure is associated both to the construction of individual perceptions about the organization as well as to emergent interactive behaviors.

------- Insert Table 1 about here --------

In Hypotheses 1 and 2 we proposed that individual polychronicity and the perception of the organization's polychronicity, respectively, were positively related to centrality. For 
each dependent variable (degree communication and indegree advice giving) we included four models: one with the control variables (model 1), one with the control variables and individual polychronicity (model 2), one with the control variables and organizational polychronicity (model 3), and one full model (model 4). We computed the VIF (Variance Inflation Factor) for all models and found that values were less than 2, indicating that multicollinearity was not a problem (Cohen et al., 2003). Table 2 reports our results.

Insert Table 2 about here

With regard to the control variables, in the final models (models 4), the number of colleagues (i.e. the number of different co-workers formally collaborating in the same projects) is significantly related to the Degree Communication and the Indegree Advice Giving. Additionally, location was significantly related to centrality in both networks, suggesting that those at the headquarters were more central in terms of communication and knowledge sharing than their offshore colleagues. Finally, also the professionals who have been with the organization for a longer number of years were more central in terms of offering advice and help related to work.

The relationship between individual polychronicity and centrality in instrumental networks

We hypothesized that individual polychronicity would be significantly related to centrality in instrumental networks. Table 2 shows that in models 2 , the coefficient for individual polychronicity was significant and positive for the degree communication $(\beta=$ $3.72 ; \mathrm{p}<0.001)$, and the indegree advice giving $(\beta=1.84 ; \mathrm{p}<0.01)$, thus providing support for Hypothesis 1. As a post-hoc analysis, we also ran the same models with the outdegree advice giving as dependent variable, but we did not find significant results. 
The interviews with our informants largely support these results. Specifically, about $40 \%$ of our interviewees expressed their preference to be polychronic, by stating that they preferred to move between different tasks, activities, and projects within the same day and felt that this was the way things should be done. For instance, William, ${ }^{1}$ told us:

I do not dislike at all doing many things at once. I have always been accustomed to do many different things, since my experience with my very first boss, who taught me the basis of everything I know now. Doing many things has always been in line with my mental capabilities. [...] Maybe it's only my mind that works like that, but I think it should be like that for others, too. If I have a problem and I think about that all day, I do not find a solution. If I do other, I think about other things, then I wake up and here it is a good idea! To sum up, I do not have problems in doing many things at once, you just need to find an equilibrium, you do some training as in every other thing in life and you do it.

Luke, a polychronic engineer narrated to us how his preference for working on various tasks simultaneously made him comfortable with squeezing relational activities, even if unscheduled, into existing work patterns:

No, it is not hard for me to interrupt and pay attention to unscheduled events and interactions. Actually, I would say that I find myself comfortable with it. [...] As a matter of fact, I interact more or less with everybody. I had to put effort at the beginning because, as you can imagine, you also need to cross the functional 'silos'. Now I communicate regularly with all the technical areas of the company. It is not

\footnotetext{
${ }^{1}$ We gave fictitious names to our informants to maintain their confidentiality.
} 
that they necessarily always open their arms to me [laughing]... But I was able to develop 'mates' in every office and I go to them and I thrive through this multitasking scenario.

The remaining interviewees, on the other hand, 'would prefer not moving from the desks, do not interact continuously with clients and colleagues, and prefer spending more time in designing in front of their computers'. Such was the case of Giacomo, who told us that he preferred working on projects that were rigidly scheduled, so that he could adhere to schedules without having to attend to events often related to colleagues' interactions that were driving his attention away from the same project:

[Name of a client company] is perfect in terms of time management! That's my dream situation. They follow a plan, which is defined in extreme detail and is followed with extreme accuracy. This means that I can stick to schedule and have a lot of time to work uninterrupted, which I prefer to the continuous interruptions that I receive in other projects.

\section{The relationship between perceived organizational polychronicity and centrality in instrumental networks}

Models 3 test the effects of perception of organizational polychronicity on the instrumental network centrality, indicating support for Hypotheses 2. The coefficients were positive and significant for the degree communication $(\beta=3.69 ; \mathrm{p}<0.001)$ and the indegree advice giving $(\beta=1.35 ; \mathrm{p}<0.05)$. As a post-hoc analysis, we also ran the same model with the outdegree advice giving as dependent variable, but we did not find significant results. 
Ciro, the VP of R\&D, when asked to comment about Re.Search's demands for working on multiple tasks simultaneously, explained that 'work scheduling is driven by the client. We have many international clients - big names - and don't want to say no to anyone. So, my guys are expected to work on 100 things at the same time.' This top management discourse is acknowledged by some of our informants, like Diego and Claude, who commented on their perceptions of the organizational polychronicity:

Even if we were working, let's say, on 6 projects, we could keep going with the first one, than the second, than the third and so on. But this is not what happens in Re.Search which prefers us to manage 'millions' of things simultaneously (Diego). The company is facing the fact that the market has grown exponentially and we are probably understaffed. So, not only we have many tasks we need to accomplish in a single workday [...] When we receive external requests, whatever things we are doing, we have to leave it and address the issues and quickly go back to the previous one. This is considered the normal deployment of our work and we need to adapt (Claude).

Our qualitative evidence suggests that perceiving Re.Search as an organization that asks its employees to work on multiple tasks simultaneously is related to the numerous interactions that individuals enact in the workplace, and, as a consequence, individuals' network positions. In particular, those informants who perceive the environment as polychronic suggest that they engage in more relationships because frequent task switching entails higher fragmentation of work activities that calls for subsequent integration. For instance, William and Francesco commenting on their pattern of communication interactions told us respectively: 
Re.Search wants me to work on so many different projects that I definitely need others for whatever I do... As an example, there is a person I can communicate for building up the manual, and another person to ask to for applications, because by myself I wouldn't be definitely capable of dealing with so many heterogeneous issues. I ended up building a lot of valuable relationships, also outside of Re.Search. Most of the time, with these friendly relationships I can do a lot.

In this organization we have a certain amount of resources and this is why we are on so many different projects. We are asked to continuously shift from one to another and to interrupt our work. It often happens that I am asked to give a person some information and I have to attend the requests I receive, which makes me change my task and return to that only after some time.

\section{Differentiating the effects of individual and organizational polychronicity on centrality in communication and advice networks}

When both individual polychronicity and the perception of organizational polychronicity are used in models 4, we did not find support for Hypothesis 3. In the communication network (Table 2), the coefficient of perception of organizational polychronicity is positive and significant $(\beta=2.54$; $p<0.05)$, but the coefficient of individual polychronicity is also significant and slightly higher $(\beta=2.63 ; \mathrm{p}<0.05)$. In the advice giving network only the effect of individual polychronicity is significant $(\beta=1.55 ; \mathrm{p}<0.05)$, while organizational polychronicity no longer is. As a post-hoc analysis, we also ran the same model with the outdegree advice giving as dependent variable, but we did not find significant results. 
The results of the quantitative analysis suggest that, in addition to the 'required' network represented by the number of colleagues individuals are co-staffed in the same projects, only individual polychronicity is associated to centrality in the advice network, i.e. in interactions focused on feedback exchange and suggestion-giving and thus characterized by knowledge sharing. Both individual polychronicity and organizational polychronicity are associated to centrality in the communication network, which captures, more generically, any work-related interaction. We looked for further explanations of these findings in our qualitative data.

When it comes to knowledge intensive content, such as advice exchanges, people seem to prefer to enact their personal preferences in terms of doing multiple tasks at the same time and, in order to do so, perform specific strategies related to managing interactions with others. In our data this is particularly evident with monochronic individuals, who shield themselves from having 'too many interactions'. Examples are given by informants telling us that they 'close the door' of their offices or they 'hide' at their desks to avoid too many requests for help. A similar example is given by Giacomo, when he tells us that sometimes he prefers not to respond to emails asking for suggestions and advice.

There is this colleague who calls me three times a day. What does he want from me? Some drawings and some help to figure out what they mean. But he goes on... Then he sends me emails for further explanations. I tell him to contact the project leader, but he continues. [....] That is over-communication and I cannot stand it, so I stop responding to his emails and that's it. 
While it is not possible to avoid all the formal communications employees are required to be part of by the organization, the above evidence suggests that when it comes to knowledge intensive interactions personal preferences may prevail.

\section{Discussion}

The purpose of this study was to investigate the existence of positive relationships between individual polychronicity and perceptions of organizational polychronicity and individual positions in instrumental networks like the communication and the advice networks. We found support for our predictions. We also argued that when analyzed together, the perceptions of organizational norms (perceived organizational polychronicity) would have a higher relative importance than individual preferences (individual polychronicity). This prediction was not supported; rather, our results demonstrated a stronger role of individual polychronicity in relation to instrumental networks centrality. Our study makes three main contributions.

First of all, we provide insights to the debate currently ongoing in the social network literature about the prevalence of structure versus individual characteristics and cognitions in affecting the individuals' positions in a network (e.g. Kilduff and Brass, 2010; Tasselli et al., 2015). A structuralist tradition argues that the characteristics of people's networks (e.g. sparse or dense networks) drive the evolution of individuals' networks and individual-level outcomes (like reputation, innovativeness, job opportunities, e.g Rodan and Galunic, 2004; Uzzi and Spiro, 2005), overlooking how the same people may express their choices or be guided by their preferences, beliefs, and values in social actions. Emirbayer and Goodwin (1994) and Emirbayer and Mische (1998) have criticized this position, arguing that even within the constraints of structure or broader cultural categories, actors can try to structure their social order, according to their values, beliefs, preferences, and commitments. Recently, 
Bensaou and colleagues (2014) offered a relevant contribution to the debate by studying the level of agency behind different networking strategies adopted by groups of service professionals navigating role transitions. The authors show that the development of such strategies cannot be explained only by prior network structure (network size and density). Conversely, taking into account the ways in which professionals draw on cultural categories like their moral codes, professional schema, locus of influence and time schema (orientation to the past, the present or the future), provides a deeper understanding of the nuances in networking behaviors.

Our study enriches this debate by disentangling the effects of individual preferences, organizational constraints, and individual interpretations of the organization in individuals' social networks. In analyzing our relationships, we controlled for the number of people the individuals were collaborating on projects at that time. Consequently, we were able to understand who the individuals were required to work with (i.e. required ties) and who they had the choice to work with (i.e. voluntary ties). Put differently, after stripping away the required network, it is reasonable to assume that everyone remaining in the person's network is a voluntary tie, and this is where we expected individual characteristics and perceptions of organizational norms would play a major role. As expected, in both the communication and advice networks, the constraints imposed by the organization in terms of formal assignment to teams are significantly related to centrality. However, we display that beyond organizational requirements, individual preferences and dispositions shape individuals' position in both networks, supporting the perspective that even within the organizational constraints that bound actions, people are guided by individual preferences and perceptions. Our treatment of organizational polychronicity, that draws on Swidler's theory of culture as a tool kit, disentangles another process at play. In contrast to previous studies (e.g. Slocombe and Bluedorn, 1999) we suggest that even within the same work unit, individuals may 
experience some variability in terms of their perception of organizational polychronicity as a consequence of drawing differently from the cultural tool kits proposed to them. Thus, perceived organizational temporal norms provide some constraints but they also enable actors some degree of choice in enacting their social world.

We find that the perception of organizational polychronicity plays a role comparable to the individual polychronicity (but not higher as we hypothesized) in the communication network but stops being significant in the advice network. We believe that the elucidation of these findings requires to unpack the differences between the two types of networks that we measured. While both are instrumental networks, related to actions undertaken to accomplish work, the advice network rests on transmitting help, support, orientation, as well as professional values (Gibbons, 2004). Advice relations can be considered forms of knowledge transfer within organization (e.g. Lomi et al. 2014). Given these characteristics, advice networks likely require larger investments in terms of time, energy and efforts to develop and maintain as compared to communication, triggering concerns in people about their possibility and opportunity to deal with such relationships. Thus, professionals, although constrained by what the organization formally imposes them to do (e.g. how many people they are assigned to work with), may be driven by their individual preferences and beliefs about what is the best way to work - and the subsequent behaviors that best suit them - more than by what they perceive is the organizational cultural norm. Stated differently, when it comes to the 'core' of their work as professionals (i.e. knowledge sharing) individual preferences matter and make the difference in how people behave. Overall our findings are relevant because they testify how different factors, potentially pointing to different directions, are at play in shaping individuals' network positions (see also Jonczyk et al. 2016). Individual preferences and dispositions could in fact be misaligned with organizational requirements or the perceptions of organizational norms (such as in the case of a monochronic individual perceiving that the 
organization prefers people to be engaged in multiple tasks simultaneously or a polychronic individual perceiving that the organization demands people to attend single activities at a time). Our study suggests that individuals could be eventually pushed into network patterns in different directions from what organizations prefer and value, even at their own disadvantage (e.g. being perceived as less compliant, with potential negative consequences in terms of appraisal, future work assignment, and career advancements). We thus propose that managers should not underestimate the importance of fit between individual preferences and interpretations of organizational norms in promoting behaviors that are consistent with organizational aims.

Secondly, we advance research on time and polychronicity by providing evidence on previously unexplored correlates of individual and perceptions of organizational polychronicity, adding to the budding literature that aims to understand how individual timerelated variables affect behaviors in the workplace (e.g. Kaufman-Scarborough and Lindquist, 1999; Madjar and Oldham, 2006; Souitaris and Maestro, 2010). When theorizing about the influence of time-related preferences like polychronicity, previous research (e.g. Hall, 1983; Kaufman-Scarborough and Lindquist, 1999) refers to interpersonal relationships but do not theorize or measure whether individual polychronicity and perceived organizational polychronicity relate to workplace social networks. Given that one of the most important vehicles through which communication and knowledge flow in organization are social networks via the positions individuals occupy in it (Cross and Cummings, 2004; Tagliaventi and Mattarelli, 2006), and given that individuals' sense of time acts both as enabler and constraint of people's communication acts (Ballard and Seibold, 2000), our study is an important step in understanding the constitutive relationship between time and communication. 
Finally, investigating how individual polychronicity and the individuals' perceptions about the preferences of the organization affect social networks integrates micro variables and macro-level structures such as the organizational social networks. While research on timing issues and variables at the individual level is quite developed in terms of antecedents and consequences, some authors have called for more studies that integrate different levels of analysis because 'time-oriented behaviors of individuals have the potential to affect outcomes at both the group and organizational levels and the study of such dynamics may be a promising expansion of inquiry at all levels' (Waller et al., 1999: 255). Also Perlow (1999) lamented the absence of studies that link individual perceptions of time with social constructions and with social networks. We therefore respond to these calls showing that professionals' perceptions of the temporal norms of their organization make a difference in terms of how much they are willing to give and take in terms of communication influencing overall the amount of information that flows between individuals, groups, and the organization at large.

\section{Limitations and future research directions}

While we were interested in understanding how individuals' preferences for engaging in multiple tasks simultaneously interact with their beliefs about the organization's preferences to manifest in networking behaviors, we acknowledge that preferences and perceptions about the organization are not static, and that social networks change. Future research is needed to better comprehend not only the causal direction of the relationships among time-related individual preferences and network position but especially how they co-evolve over time (Mund and Neyer, 2014; Tasselli et al., 2015).

In this paper we did not delve into how people sequenced their tasks over time, but only on their preferences for engaging in multiple tasks simultaneously. Investigating the 
sequencing behaviors of individuals, e.g. between expected and unexpected events, between different working spheres, between quiet time and interactive activities (e.g. Perlow, 1999; Gonzales and Mark, 2004) in relation to preferences and social networks is another avenue for future research.

In addition, we conducted our work in a single organization and with a limited number of respondents. Even though our evidence may not be generalized to other settings, the access that we were able to gain in this context enabled us to investigate organizational actors deeper, via the collection and analysis of qualitative evidence that helped us to better understand and explain our quantitative results. However, to investigate the relationships between individual polychronicity, the perception of the organizational polychronicity and social networks' centrality it is necessary to consider other contexts and organizations. Re.Search presented some peculiarities that we need to take into account. First, Re.Search was a highly prestigious organization that provided $R \& D$ professionals with the opportunity to work on cutting-edge innovation projects, triggering in most of them a high level of loyalty. What would happen in organizations perceived as less prestigious? How would professionals negotiate their individual preferences and the organizational preferences in less innovative and dynamic contexts?

Second, the organization we studied staffed R\&D members on a large number of projects in the same time framework, and asked them to work on multiple tasks and activities simultaneously, prompting to higher levels of organizational polychronicity. However, the mean level of perceived organizational polychronicity was 4.59 with a standard deviation of 1.29, which suggests that even though the organization was perceived on average above the central level of the scale there was variation that is consistent with our cultural tool kits perspective. Nevertheless, an interesting avenue for future research would lie in investigating organizational contexts differing in the temporal tool kits that they offer to their employees 
(such as organization coordination requirements, feedback cycles, and workplace collaborative technology, Ballard and Seibold, 2003; Ballard and Seibold, 2004), and thus unravelling how organizational polychronicity interacts with other variables.

Related to this latter comment, in our paper we propose a cultural tool kit perspective on organizational polychronicity, but we do not explore what makes people develop different perceptions of the time related organizational demands. In our results section we point to how organizational polychronicity is positively correlated with the number of colleagues a person is required to work with and to individual polychronicity. These results suggest that the formal structures provided by the organization (i.e. how people are staffed in different projects) and the individual preferences can be related to how perceptions about organizational polychronicity are formed. However, future research should further explore how perceptions of organizational polychronicity are formed, i.e. its antecedents. To this regard, we hope that the tool kit perspective that we propose as an anchoring framework will lead to a new way to look at polychronicity in organizations.

\section{Funding}

This research was supported by funding from MIUR (Italian Ministry of Research) through the PRIN project 'International Sourcing of Knowledge: Organizational Factors and System Effects' (grant number: 2007CFABCS). 


\section{References}

Ahuja, M.K., D.F. Galletta, \& K.M. Carley. (2003). Individual Centrality and Performance in Virtual R\&D Groups: An Empirical Study. Management Science, 49(1), 21-38.

Arndt, A., T.J Arnold, \& T.D. Landry. (2006). The effects of polychronic orientation upon retail employee satisfaction and turnover. Journal of Retailing, 82, 319-330.

Asendorpf, J. B., \& Wilpers, S. (1998). Personality effects on social relationships. Journal of Personality and Social Psychology, 74(6), 1531-1544.

Ashforth, B. E., Kreiner, G. E., \& M. Fugate. (2000). All in a day's work: Boundaries and micro role transitions. Academy of Management Review, 25(3), 472-491.

Baldwin, T. T., M.D. Bedell, \& J.L. Johnson. (1997). The social fabric of a team-based M.B.A. program: Network effects on student satisfaction and performance. Academy of Management Journal, 40, 1369-1397.

Ballard, D. I., \& D.R. Seibold. (2000). Time orientation and temporal variation across work groups: Implications for group and organizational communication. Western Journal of Communication, 64, 218-242.

Ballard, D. I., \& Seibold, D. R. (2003). Communicating and organizing in time: A meso level model of organizational temporality. Management Communication Quarterly, 16, 380-415.

Ballard, D. I., \& Seibold, D. R. (2004). Communication-related organizational structures and work. Communication Monographs, 71(1): 1-27.

Bensaou, B. M., Galunic, C., \& C. Jonczyk-Sedes. (2014). Players and purists: Networking strategies and agency of service professionals. Organization Science, 25: 29-56.

Bertolotti, F., D.M. Macrì, \& M.R. Tagliaventi. (2005). Spontaneous self-managing practices: Evidence from the field. Journal of Management Inquiry, 14(4), 1-19.

Bertolotti, F., \& M.R.Tagliaventi. (2007). Discovering complex interdependencies in organizational settings: the role of social network analysis in qualitative 
research. Qualitative Research in Organizations and Management: An International Journal, 2(1), 43-61.

Blount, S. \& G.A. Janicik. (2001). When plans change: Examining how people evaluate timing changes in work organizations. Academy of Management Review, 26(4) 566-585.

Blount, S. \& S. Leroy. (2007). Individual temporality in the workplace: how individuals perceive and value time at work. Research in the sociology of work, 17, 147-177.

Bluedorn, A.C., T.J. Kalliath, M.J. Strube, \& G.D. Martin. (1999). Polychronicity and the inventory of polychronic values: The development of an instrument to measure a fundamental dimension of organizational culture. Journal of Managerial Psychology, 14(3/4), 205-230.

Bluedorn, A.C., C.F. Kaufman, \& P.M. Lane. (1992). How many things do you like to do at once? An introduction to monochronic and polychronic time. Academy of Management Executive, 6(4), 17-26.

Bluedorn, A.C. (2002). The Human Organization of Time: Temporal Realities and Experience. Stanford University Press, Stanford, CA.

Borgatti, S.P., M.G. Everett, \& L.C. Freeman. (2002). Ucinet for Windows, version 6.0: Software for social network analysis. Harvard: Analytic Technologies.

Brass, D.J. (1984). Being in the Right Place: a Structural Analysis of Individual Influence in an Organization. Administrative Science Quarterly, 29, 518-539.

Brass, D. J., Galaskiewicz, J., Greve, H. R., \& W. Tsai. (2004). Taking stock of networks and organizations: A multilevel perspective. Academy of Management Journal, 47(6), 795-817.

Casciaro, T., Barsade, S. G., Edmondson, A. C., Gibson, C. B., Krackhardt, D., \& G. Labianca. (2015). The Integration of Psychological and Network Perspectives in Organizational scholarship. Organization Science, 26(4), 1162-1176. 
Cohen, J. (1960). A coefficient of agreement for nominal scales. Educational and psychological measurement, 20(1), 37-46.

Cohen, J., Cohen, P., Aiken, L. S., \& S.G. West. (2003). Applied multiple regression/Correlation analysis for the behavioral sciences $\left(3^{\text {rd }} \mathrm{ed}\right)$. Erlbaum, Mahwah, NJ.

Conte, J.M. \& J.N. Gintoft. (2005). Polychronicity, big five personality dimensions, and sales performance. Human Performance, 18, 427-444.

Conte, J. M. \& R.R. Jacobs. (2003). Validity evidence linking polychronicity and big five personality dimensions to absence, lateness, and supervisory performance ratings. Human Performance, 16, 107-129.

Cotte, J., \& S. Ratneshwar. (1999). Juggling and hopping: What does it mean to work polychronically? Journal of Managerial Psychology, 14 (3/4), 184-204.

Creswell, D.J. (2003). Research design: Qualitative, quantitative, and mixed methods approaches. Sage, New York.

Cross, R. \& J.N. Cummings. (2004). Tie and network correlate of individual performance in knowledge-intensive work. Academy of Management Journal, 47(6), 928-937.

Day, D. V. \& M. Kilduff (2003). Self-monitoring personality and work relationships: Individual differences in social networks. M. R. Barrick, A. M. Ryan, eds. Personality and work: Reconsidering the role of personality in organizations. Jossey-Bass, San Francisco, $205-228$.

Emirbayer, M., \& J. Goodwin. (1994). Network analysis, culture, and the problem of agency. American journal of sociology, 99(6), 1411-1454.

Emirbayer, M., \& A. Mische. (1998). What is agency? American journal of sociology, 103(4), 962-1023. 
Fang, R., Landis, B., Zhang, Z., Anderson, M. H., Shaw, J. D., \& M. Kilduff. (2015). Integrating Personality and Social Networks: A Meta-Analysis of Personality, Network Position, and Work Outcomes in Organizations. Organization Science, 26(4): 1243-1260.

Freeman, L.C. (1979). Centrality in social networks: I. Conceptual clarification. Social Networks, 1, 215-239.

Giorgi, S., Lockwood, C., \& M. A. Glynn. (2015). The many faces of culture: Making sense of 30 years of research on culture in organization studies. The academy of Management Annals, 9 (1), 1-54.

Gibbons, D. E. (2004). Friendship and advice networks in the context of changing professional values. Administrative Science Quarterly, 49(2), 238-262.

Gobbo, F., \& M. Vaccari. (2008). The pomodoro technique for sustainable pace in extreme programming teams. International Conference on Agile Processes and Extreme Programming in Software Engineering, Springer, Berlin, Heidelberg , pp. 180-184.

González, V. M., \& G. Mark. (2004). Constant, constant, multi-tasking craziness: Managing multiple working spheres. In E. Dykstra-Erickson \&M. Tscheligi (Eds.), Proceedings of the 2004 conference on Human factors in computing systems (CHI’04) (pp. 113-120). New York: ACM.

Granovetter, M. (1985). Economic action and social structure: The problem of embeddedness. American journal of sociology, 91(3), 481-510.

Gubrium, J.F. \& J.A. Holstein. (2003). Handbook of interview research: Context \& method. Sage, Thousand Oakes.

Hall, E.T. (1959). The Silent Language. Fawcett Publications, New York. Hall, E.T. (1983). The Dance of Life: The Other Dimension of Time. Anchor Press, New York. 
Hall, E.T. \& M.R. Hall. (1990). Understanding cultural differences. Intercultural Press, Yarmouth, ME.

Hanneman, Robert A. \& M. Riddle. (2005). Introduction to social network methods.

Riverside, CA: University of California, Riverside (published in digital form at http://faculty.ucr.edu/ hanneman/ )

Hecht, T. D. \& J.A. Allen. (2005). Exploring links between polychronicity and well-being from the perspective of person-job fit: Does it matter if you prefer to do only one thing at a time? Organizational Behavior and Human Decision Processes 98 155-178.

Hui, C., S.K. Lam, \& K.K.S. Law. (2000). Instrumental values of organizational citizenship behavior for promotion: A field quasi-experiment. Journal of Applied Psychology, 85(5), 822-828.

Ibarra, H. (1992). Homophily and differential returns: Sex differences in network structure and access in an advertising firm. Administrative Science Quarterly, 422-447.

Ibarra, H. (1993). Network centrality, power, and innovation involvement: Determinants of technical and administrative roles. Academy of Management Journal, 36, 471-501.

Jang, J. \& R.T. George. (2012). Understanding the influence of polychronicity on job satisfaction and turnover intention: A study of non-supervisory hotel employees. International Journal of Hospitality Management, 31, 588-595.

Jonczyk, C. D., Y.G. Lee, C.D. Galunic, \& B.M. Bensaou. (2016). Relational changes during role transitions: The interplay of efficiency and cohesion. Academy of Management Journal, 59(3), 956-982.

Judge, T. A., \& C.P. Zapata. (2015). The person-situation debate revisited: Effect of situation strength and trait activation on the validity of the Big Five personality traits in predicting job performance. Academy of Management Journal, 58(4), 1149-1179. 
Kalish, Y. \& G. Robins. (2006). Psychological predispositions and network structure: The relationship between individual predispositions, structural holes and network closure. Social Network, 28 (1), 56-84.

Kaufman, C.F., P.M. Lane, \& J.D. Lindquist. (1991). Exploring more than 24 hours a day: A preliminary investigation of polychronic time use. Journal of Consumer Research, 18 (3), $392-401$.

Kaufman-Scarborough, C. \& J.D. Lindquist. (1999). Time management and polychronicity: Comparisons, contrasts, and insights for the workplace. Journal of Managerial Psychology $14288-312$

Kellogg, K. C. (2011). Hot lights and cold steel: Cultural and political toolkits for practice change in surgery. Organization Science, 22(2), 482-502.

Kilduff, M., \& D.J. Brass. (2010). Organizational Social Networks Research: Core Ideas and Key Debates. The Academy of Management Annals, 4(1), 317-357.

Kilduff, M. \& W. Tsai. (2003). Social Networks and Organizations. Sage, Thousand Oaks.

Klein, K. J., B.-C. Lim, J.L. Saltz, \& D.M. Mayer. (2004). How Do They Get There? An Examination of the Antecedents of Centrality in Team Networks. Academy of Management Journal, 47, 952-963.

Kleinbaum, A.M., Jordan, A.H., \& P.G. Audia. (2015). An Altercentric Perspective on the Origins of Brokerage in Social Networks: How Perceived Empathy Moderates the SelfMonitoring Effect. Organization Science, 26(4), 1226 - 1242.

König, C. J., Bühner, M., \& G. Mürling. (2005). Working memory, fluid intelligence, and attention are predictors of multitasking performance, but polychronicity and extraversion are not. Human Performance, 18, 243-266.

König, C.J. \& M.J. Waller. (2010). Time for Reflection: A Critical Examination of Polychronicity. Human Performance, 23, 173-190. 
Krackhardt, D. \& J.R. Hanson. (1993). Informal networks: The company behind the chart. Harvard Business Review, 104-111.

Landis, B. (2016). Personality and social networks in organizations: A review and future directions. Journal of Organizational Behavior, 37, S107-S121.

Lee, Y. H., Yang, L. S., Wan, K. M., \& G.H. Chen. (2010). Interactive effects of personality and friendship networks on contextual performance. Social Behavior and Personality: an international journal, 38(2), 197-208.

Lewis, K., Kaufman, J., Gonzalez, M., Wimmer, A., \& N. Christakis. (2008). Tastes, ties, and time: A new social network dataset using Facebook. com. Social Networks, 30(4), 330-342.

Liu, Y., \& M. Ipe. (2010). How do they become nodes? Revisiting team member network centrality. The Journal of psychology, 144(3), 243-258.

Lomi, A., D. Lusher, P.E. Pattison, \& G. Robins. (2013). The focused organization of advice relations: A study in boundary crossing. Organization Science, 25(2), 438-457.

Lucas, R. E., \& Donnellan, M. B. (2009). If the person-situation debate is really over, why does it still generate so much negative affect?. Journal of Research in Personality, 43(2), 146-149.

Madjar, N. \& G.R. Oldham. (2006). Task rotation and polychronicity: Effects on individuals' creativity. Human Performance, 19, 117-131.

Mattarelli, E., \& M.R. Tagliaventi. (2010). Work-related identities, virtual work acceptance and the development of glocalized work practices in globally distributed teams. Industry and Innovation, 17(4), 415-443.

Mattarelli, E., F. Bertolotti, \& V. Incerti. (2015). The interplay between organizational polychronicity, multitasking behaviors and organizational identification: A mixed-methods study in knowledge intensive organizations. International Journal of Human-Computer Studies, 79, 6-19. 
Mehra, A., M. Kilduff, \& D.J. Brass. (2001). The social networks of high and low selfmonitors: Implications for workplace performance. Administrative Science Quarterly, 35, $121-146$.

Meyer, R. D., R.S. Dalal, \& R. Hermida. (2010). A review and synthesis of situational strength in the organizational sciences. Journal of Management, 36: 121-140.

Miles, M.B. \& A. Huberman. (1994). Qualitative data analysis: an expanded sourcebook. Sage Publications, Beverly Hills, CA.

Mohammed, S. \& D. Harrison. (2013). The clocks that time us are not the same: A theory of temporal diversity, task characteristics, and performance in teams. Organizational Behavior and Human Decision Processes, 122(2), 244-256.

Mund, M. \& F. J. Neyer. (2014). Treating personality-relationship transactions with respect: Narrow facets, advanced models, and extended time frames. Journal of Personality and Social Psychology, 107(2), 352-368.

Oh, H. \& M. Kilduff. (2008). The Ripple Effect of Personality on Social Structure: SelfMonitoring Origins of Network Brokerage, Journal of Applied Psychology, 93(5), 1155 1164.

O'Leary, M.B., M. Mortensen, \& A.W. Woolley. (2011). Multiple Team Membership: A Theoretical Model of its Effects on Productivity and Learning for Individuals and Teams. The Academy of Management Review, 36(3), 461-478.

Pappas, J. M. \& B. Wooldridge. (2007). Middle Managers' Divergent Strategic Activity: An Investigation of Multiple Measures of Network Centrality. Journal of Management Studies, 44, 323-341. 
Payne, S. C. \& J. Philo. (2002). Identifying those who prefer to do more with less. Paper presented at the 17th annual convention of the Society of Industrial and Organizational Psychology, Toronto, Canada.

Perlow, L.A. (1999). The time famine: Toward a sociology of work time. Administrative Science Quarterly, 44, 57-81.

Pollet, T. V., Roberts, S. G. B., \& R. I. M. Dunbar. (2011). Extraverts have larger social network layers. Journal of Individual Differences, 32(3), 161-169.

Pratt, M. G., K.W. Rockmann, \& J.B. Kaufmann. (2006). Constructing Professional Identity: The Role of Work and Identity Learning Cycles in the Customization of Identity Among Medical Residents. Academy of Management Journal, 49(2), 235-262.

Rodan, S., \& C. Galunic. (2004). More than network structure: How knowledge heterogeneity influences managerial performance and innovativeness. Strategic Management Journal, 25(6), 541-562.

Sykes, T.A, V. Venkatesh, \& J. L. Johnson. (2014). Enterprise system implementation and employee job performance: Understanding the role of advice networks. MIS Quarterly 38(1) 51-72.

Shrader, C. B., Lincoln, J. R., \& A. N. Hoffman. (1989). The network structures of organizations: Effects of task contingencies and distributional form. Human Relations, 42(1), 43-66.

Slocombe, T. \& A.C. Bluedorn. (1999). Organizational behavior implications of the congruence between preferred polychronicity and experienced work-unit polychronicity. Journal of Organizational Behavior 20 75-99.

Soutaris, V., \& M. Maestro. (2010). Polychronicity in top management teams: the impact on strategic decision processes and performance of new technology ventures. Strategic Management Journal 31 652-678. 
Sparrowe, R.T., R.C. Liden, S.J. Wayne, \& M.L. Kraimer. (2001). Social network and the performance of individuals and groups. Academy of Management Journal, 44(2), 316-325.

Stephens, K. K., Cho, J. K., \& D. I. Ballard. (2012). Simultaneity, sequentiality, and speed: Organizational messages about multiple task completion. Human Communication Research, 38(1), 23-47.

Swidler, A. (1986). Culture in action: Symbols and strategies. American Sociological Review, $51,273-286$.

Swidler, A. (2000). Cultural power and social movements. In Culture and Politics (pp. 269283). Palgrave Macmillan, New York.

Tagliaventi, M.R. \& E. Mattarelli. (2006). The role of networks of practice, value sharing, and operational proximity in knowledge flows between professional groups. Human Relations, 59, 291-319.

Tasselli, S., Kilduff, M., \& J.I. Menges. (2015). The Microfoundations of Organizational Social Networks A Review and an Agenda for Future Research. Journal of Management, 41(5), 1361-1387.

Carton, G., \& P. Ungureanu (2018). Bridging the Research-Practice Divide: A Study of Scholar-Practitioners' Multiple Role Management Strategies and Knowledge Spillovers Across Roles. Journal of Management Inquiry, 27 (4), 436-453.

Uzzi, B., \& J. Spiro. (2005). Collaboration and creativity: The small world problem. American Journal of Sociology, 111(2), 447-504.

Van Scotter, J., S.J. Motowidlo, \& T.C. Cross. (2000). Effects of task performance and contextual performance on systemic rewards. Journal of Applied Psychology, 85(4), 526535. 
Waller, M.J., R.C. Giambatista, \& M.E. Zellmer-Bruhn. (1999). The effects of individual time urgency on group polychronicity. Journal of Managerial Psychology, 14(3/4), 244256.

Wasserman, S. \& K. Faust. (1994). Social network analysis: Methods and applications. Cambridge University Press, Cambridge, MA.

Wasson, C. (2004). Multitasking during virtual meetings. Human Resource Planning, 27(4), $47-61$.

Weber, K. (2005). A toolkit for analyzing corporate cultural toolkits. Poetics, 33(3-4), 227252.

Wrzesniewski, A., \& J. E. Dutton. (2001). Crafting a Job: Revisioning Employees as Active Crafters of Their Work. Academy of Management Review, 26(2), 179-201. 


\section{Appendix A: Scale items used to measure polychronicity}

Individual Polychronicity (Kaufman et al., 1991; Bluedorn et al., 1992)

I do not like to juggle several activities at the same time

People should not try to do many activities at once

When I sit down at my desk, I work on one activity at a time

I am comfortable doing several activities at the same time

Perception of organizational Polychronicity (Bluedorn et al., 1992, 1999; Souitaris and Maestro, 2010)

In [Organization] we like to juggle several activities at the same time.

In [Organization] we would rather complete an entire activity everyday than complete parts of several activities. (Reverse-scored)

In [Organization] we believe people should try do many activities at once.

In [Organization] when we work by ourselves we usually work on one activity at a time. (Reverse-scored)

In [Organization] we prefer to do one activity at a time. (Reverse-scored) 
Table 1: Descriptive statistics and correlations ${ }^{a}$

\begin{tabular}{|c|c|c|c|c|c|c|c|c|c|c|}
\hline Variables & Mean & SD & 1 & 2 & 3 & 4 & 5 & 6 & 7 & 8 \\
\hline 1. Organizational tenure & 4.75 & 5.58 & & & & & & & & \\
\hline 2. Position & .27 & .43 & .00 & & & & & & & \\
\hline 3. Location & .82 & .39 & .03 & $\begin{array}{l}- \\
.26^{*}\end{array}$ & & & & & & \\
\hline 4. Number of colleagues & 23.03 & 16.73 & .16 & .05 & $.36^{* *}$ & & & & & \\
\hline 5. Project complexity & 2.23 & .37 & -.14 & -.18 & .08 & $.26^{*}$ & & & & \\
\hline 6. Individual polychronicity & 4.01 & 1.28 & -.12 & .19 & .08 & .06 & -.07 & & & \\
\hline $\begin{array}{l}\text { 7. Perception of organization's } \\
\text { polychronicity }\end{array}$ & 4.59 & 1.29 & -.03 & .08 & .19 & $.26^{*}$ & .08 & $.45^{* *}$ & & \\
\hline 8. Degree communication & 26.73 & 14.28 & .13 & .04 & $.45^{* *}$ & $.47^{* *}$ & .09 & $.38^{* *}$ & $.47^{* *}$ & \\
\hline 9. Indegree advice giving & 8.51 & 7.52 & $.26^{*}$ & .20 & $.37^{* * *}$ & $.50^{* *}$ & .05 & $.36^{* *}$ & $.37^{* *}$ & $.72^{* *}$ \\
\hline
\end{tabular}


Table 2: Regression results for degree communication and indegree advice giving ${ }^{\mathrm{a}}$

\begin{tabular}{|c|c|c|c|c|c|c|c|c|}
\hline \multirow[b]{2}{*}{ Variables } & \multicolumn{4}{|c|}{ Degree communication } & \multicolumn{4}{|c|}{ Indegree advice giving } \\
\hline & Model 1 & Model 2 & Model 3 & Model 4 & Model 1 & Model 2 & Model 3 & Model 4 \\
\hline \multicolumn{9}{|l|}{ Control variables } \\
\hline 1. Organizational tenure & .17 & .30 & .22 & .30 & $.27^{*}$ & $.33^{*}$ & $.29^{*}$ & $.33^{*}$ \\
\hline 2. Position & 3.78 & 1.57 & 2.64 & 1.44 & $4.54^{*}$ & 3.43 & $4.10^{*}$ & 3.38 \\
\hline 3. Location & $13.56^{* *}$ & $12.12^{* *}$ & $11.85^{* *}$ & $11.36^{* *}$ & $6.01^{* *}$ & $5.27^{*}$ & $5.36^{*}$ & $5.06^{*}$ \\
\hline 4. Number of colleagues & $.31^{* *}$ & $.29^{* *}$ & $.23^{* *}$ & $.24^{*}$ & $.15^{* *}$ & $.14^{* *}$ & $.13^{* *}$ & $.13^{* *}$ \\
\hline 5. Project complexity & 0.68 & 1.74 & .35 & 1.20 & .41 & .91 & .27 & .76 \\
\hline \multicolumn{9}{|l|}{ Main effects } \\
\hline 6. Individual polychronicity & & $3.72^{* * *}$ & & $2.63^{*}$ & & $1.84^{* *}$ & & $1.55^{*}$ \\
\hline $\begin{array}{l}\text { 7. Perception of organization's } \\
\text { polychronicity }\end{array}$ & & & $3.69^{* * *}$ & $2.54^{*}$ & & & $1.35^{*}$ & .68 \\
\hline$\Delta \mathrm{R}^{2 \mathrm{~b}}$ & & .10 & .10 & .04 & & .09 & .05 & .01 \\
\hline$\Delta \mathrm{F}^{\mathrm{b}}$ & & 1.78 & 1.66 & -0.13 & & 1.55 & .04 & -1.17 \\
\hline $\mathrm{R}^{2}$ & & & & .48 & & & & .48 \\
\hline $\operatorname{Adj} R^{2}$ & & & & .43 & & & & .43 \\
\hline $\mathrm{F}$ & & & & $8.38^{* * *}$ & & & & $8.45^{* * *}$ \\
\hline
\end{tabular}


Table R1: Regression results for outdegree advice givinga

\begin{tabular}{|c|c|c|c|c|}
\hline \multirow[b]{2}{*}{ Variables } & \multicolumn{4}{|c|}{ Outdegree advice giving } \\
\hline & Model 1 & Model 2 & Model 3 & Model 4 \\
\hline \multicolumn{5}{|l|}{ Control variables } \\
\hline 1. Organizational tenure & -.19 & -.16 & -.19 & -.16 \\
\hline 2. Position & -.84 & -1.40 & -.93 & -1.38 \\
\hline 3. Location & 1.12 & .74 & .99 & .80 \\
\hline 4. Number of colleagues & $.15^{*}$ & $.15^{*}$ & $.15^{*}$ & $.15^{*}$ \\
\hline 5. Project complexity & -.78 & -.53 & -.81 & -.49 \\
\hline \multicolumn{5}{|l|}{ Main effects } \\
\hline 6. Individual polychronicity & & .92 & & .10 \\
\hline $\begin{array}{l}\text { 7. Perception of organization's } \\
\text { polychronicity }\end{array}$ & & & .26 & -.17 \\
\hline$\Delta \mathrm{R}^{2 \mathrm{~b}}$ & & .03 & .00 & .00 \\
\hline$\Delta \mathrm{F}^{\mathrm{b}}$ & & -.02 & -.37 & -.33 \\
\hline $\mathrm{R}^{2}$ & & & & .17 \\
\hline Adj $R^{2}$ & & & & .08 \\
\hline $\mathrm{F}$ & & & & 1.84 \\
\hline
\end{tabular}


Fabiola Bertolotti is Associate Professor of Management at the University of Modena and Reggio Emilia, Italy. She was a visiting scholar at the University of Texas at Austin. Her research interests include the sharing of knowledge in work groups and among professional workers, and the rela- tionship between social networks and performance of teams operating in complex scenarios char- acterized by multiple team membership. Her work has appeared in journals such as Organization Studies, Academy of Management Learning and Education, Research Policy, Journal of Management Inquiry, and New Technology Work and Employment. [Email: fabiola.bertolotti@ unimore.it]

Elisa Mattarelli is Associate Professor of Management at the University of Modena and Reggio Emilia, Italy. She was a visiting scholar at Stanford University and the University of Arizona. Her research interests include team dynamics, identity processes, and collaborative technology use, with a focus on distributed and knowledge-intensive organizational contexts. Her work has appeared in journals such as Organization Science, Human Relations, Journal of Management Studies, Organization Studies, and Research Policy. [Email: elisa.mattarelli @unimore.it]

Janet Dukerich is Senior Vice Provost for Faculty Affairs and Professor in the Management Department at the McCombs School of Business. She holds the Harkins \& Company Centennial Chair at The University of Texas at Austin. Her research focuses on organizational identification and the creation and maintenance of organizational identity. Her scholarly work has appeared in Administrative Science Quarterly, Academy of Management Journal, Organizational Behavior and Human Decision Processes, Journal of Personality and Social Psychology, Journal of Applied Social Psychology, Journal of Business Ethics, and Human Relations. [Email: janet.dukerich@mccombs.utexas.edu]

\section{Corresponding Author:}

Elisa Mattarelli

Department of Engineering Science and Methods

University of Modena and Reggio Emilia

Via Amendola 2, Pad. Morselli Reggio

Emilia 42122

Italy

T: +390522 522275

elisa.mattarelli@unimore.it

\section{Other Author(s):}

Fabiola Bertolotti

Department of Sciences and Methods for Engineering

University of Modena and Reggio Emilia

Reggio Emilia

Italy

fabiola.bertolotti@unimore.it

Janet Dukerich

Management Department

McCombs School of Business

University of Texas at Austin

Austin, TX 78712

janet.dukerich@ mccombs.utexas.edu 